\title{
SEGAL'S CONJECTURE AND THE BURNSIDE RINGS OF FUSION SYSTEMS
}

\author{
ANTONIO DÍAZ AND ASSAF LIBMAN
}

\begin{abstract}
For a given saturated fusion system $\mathcal{F}$ we define the $\operatorname{ring} A(S) \mathcal{F}$ of the $\mathcal{F}$-invariants of the Burnside ring functor. We show how this ring is related to the Burnside ring of the fusion system $\mathcal{F}$ and how it appears naturally in the analogue of Segal's conjecture for the classifying spectrum $\mathbb{B} \mathcal{F}$. We give an explicit description of $A(S)^{\mathcal{F}}$ and we prove it is a local ring.
\end{abstract}

\section{INTRODUCTION}

A saturated fusion system $\mathcal{F}$ on a finite $p$-group $S$ is a small category whose objects are the subgroups of $S$. Its morphism sets $\mathcal{F}(P, Q)$ consist of group monomorphisms $P \rightarrow Q$, where $P, Q \leq S$, which are subject to a certain set of axioms listed in $\S 2$. Isomorphic objects in $\mathcal{F}$ are called $\mathcal{F}$-conjugate. Puig was the first to define these objects but in this note we will use the formulation of Broto-Levi-Oliver in [2]. The model is the category $\mathcal{F}_{S}(G)$ associated to a Sylow $p$-subgroup $S$ of a finite group $G$. In this case the objects are the subgroups of $S$ and the morphisms in $\mathcal{F}_{S}(G)$ are those monomorphisms $P \rightarrow Q$ which are restrictions of inner automorphisms of $G$.

For any contravariant functor $H: \mathcal{F} \rightarrow \mathcal{C}$ we can consider the inverse limit $\lim _{\mathcal{F}} H$. We call this limit the $\mathcal{F}$-invariants of $H(S)$ because, as it is easy to see, it consists of the elements $x \in H(S)$ such that $\varphi(x)=\psi(x)$ for every $\varphi$, $\psi \in \mathcal{F}(P, S)$ and every subgroup $P$ of $S$. We will use the suggestive notation $H(S)^{\mathcal{F}}$ to denote this inverse limit. For example, by [2, Theorem 5.8], the cohomology of the classifying space of a $p$-local finite group is isomorphic to $H^{*}\left(S ; \mathbb{F}_{p}\right)^{\mathcal{F}}$ for the obvious functor $P \mapsto H^{*}\left(P ; \mathbb{F}_{p}\right)$ which assigns to $P \leq S$ its mod- $p$ cohomology. In this paper we study the $\mathcal{F}$-invariants $A(S)^{\mathcal{F}}$ of the functor $A: \mathcal{F} \rightarrow$ Rings which maps $P \leq S$ to its Burnside ring $A(P)$. We call this ring "the ring of $\mathcal{F}$-invariant virtual $S$-sets". Clearly $A(S)^{\mathcal{F}}$ is a subring of $A(S)$ which contains the identity and therefore the standard augmentation map $\epsilon: A(S) \rightarrow \mathbb{Z}$ restricts to an augmentation epimorphism $\epsilon: A(S)^{\mathcal{F}} \rightarrow \mathbb{Z}$ whose kernel is denoted $I(S)^{\mathcal{F}}$. Proposition 3.6 and Lemma 4.2 give an alternative description of $A(S)^{\mathcal{F}}$ and $I(S)^{\mathcal{F}}$.

Recall that the Burnside ring $A(G)$ of a finite group $G$ is the Grothendieck group of the monoid $B(G)$ of the isomorphism classes of finite $G$-sets. In symbols, $A(G)=$ $\operatorname{Gr}(B(G))$. The multiplication in this ring is induced by cartesian product of $G$ sets. As an abelian group $A(G)$ is free with one basis element for each conjugacy class of subgroups of $G$. In [3] we construct the Burnside ring $A(\mathcal{F})$ of saturated

Date: June 19, 2009.

2000 Mathematics Subject Classification. 55Q55, 19A22, $20 \mathrm{C} 20$.

Key words and phrases. Fusion systems, Burnside ring.

The authors were supported by an EPSRC grant EP/D506484/1. 
fusion system $\mathcal{F}$. As in the case of finite groups, where $A(G)$ is the Grothendieck group of the monoid of the isomorphism classes of finite $G$-sets, $A(\mathcal{F})$ is defined as the Grothendieck group of the monoid of the isomorphism classes of objects in a small category which is derived from $\mathcal{F}$ and has finite coproducts and products. See $\S 2$ for details. The additive group of $A(\mathcal{F})$ is free with one basis element for every $\mathcal{F}$-conjugacy class of $\mathcal{F}$-centric subgroups of $S$, see [2, Def. 1.6].

Our first result shows the close relation between the rings $A(\mathcal{F})$ and $A(S)^{\mathcal{F}}$. We will write $A_{(p)}(S)^{\mathcal{F}}$ for the ring $\mathbb{Z}_{(p)} \otimes A(S)^{\mathcal{F}}$ and similarly $A_{(p)}(\mathcal{F})$ for $\mathbb{Z}_{(p)} \otimes A(\mathcal{F})$.

Theorem A. The underlying group of $A(S)^{\mathcal{F}}$ is free with one generator for each $\mathcal{F}$-conjugacy class of subgroups of $S$. The $\mathbb{Z}_{(p)}$-submodule of $A_{(p)}(S)^{\mathcal{F}}$ generated by the $\mathcal{F}$-invariant $S$-sets all of whose isotropy groups are non- $\mathcal{F}$-centric subgroups of $S$, forms an ideal $N$. There is a ring isomorphism $A_{(p)}(\mathcal{F}) \cong A_{(p)}(S)^{\mathcal{F}} / N$.

The product in the ring $A_{(p)}(\mathcal{F})$ is described on basis elements explicitly in $[3$, Theorem 4.6]. Thus we have a good understanding of a quotient ring of $A_{(p)}(S)^{\mathcal{F}}$. The desire to understand this ring springs from Segal's conjecture. This conjecture was proven by Carlsson and it asserts that for any finite group $G$ the stable cohomotopy group $\pi^{0}\left(B G_{+}\right)$is isomorphic to the $I$-adic completion $A(G)_{I}^{\wedge}$, where $I$ is the augmentation ideal of the Burnside ring $A(G), \pi^{*}(-)$ denotes stable cohomotopy groups and the subscript + means adding a disjoint base-point.

A variation of Segal's conjecture at the prime $p$ is proven by Ragnarsson in [8]. He extends results of May-McClure and describes $\tilde{\pi}^{0}\left(\left(B G_{p}^{\wedge}\right)_{+}\right)$where $G$ is a finite group and $\tilde{\pi}^{*}(-)$ denotes the reduced stable cohomotopy groups. He shows that this group is isomorphic to the $p$-adic completion of a quotient of the submodule of $A(G)$ generated by the $G$-sets with $p$-power isotropy. In this paper we prove an analogue of Segal's conjecture for saturated fusion systems at the prime $p$.

Any saturated fusion system $\mathcal{F}$ on a $p$-group $S$ has a natural classifying spectrum $\mathbb{B} \mathcal{F}$ constructed by Ragnarsson in [9]. It is equipped with a structure map $\sigma_{\mathcal{F}}: \mathbb{B} S \rightarrow \mathbb{B} \mathcal{F}$ where $\mathbb{B} S$ is the suspension spectrum of $B S$ and $\mathcal{F}$ can be recovered from the pair $\left(\sigma_{\mathcal{F}}, \mathbb{B} \mathcal{F}\right)[9$, Theorem $\mathrm{A}]$. When $\mathcal{F}$ has a classifying space, i.e., if there is a $p$-local finite group $(S, \mathcal{F}, \mathcal{L})$ (see [2]), then $\mathbb{B} \mathcal{F}$ is the suspension spectrum of the $p$-completed classifying space $|\mathcal{L}|_{p}^{\wedge}$. In particular, when $\mathcal{F}=\mathcal{F}_{S}(G)$ is the saturated fusion system induced by a finite group $G$, then there is a homotopy equivalence $\mathbb{B} \mathcal{F} \simeq \Sigma^{\infty}(B G)_{p}^{\wedge}$ and $\sigma_{\mathcal{F}}$ is induced from the inclusion $B S \rightarrow B G$. Conjecturally, $\mathbb{B} \mathcal{F}$ is always a suspension spectrum of some space that one can associate to the saturated fusion system $\mathcal{F}$. Throughout the symbol $\mathbb{S}$ will denote the sphere spectrum. The next result gives a description of the cohomotopy group $\pi^{0}(\mathbb{B} \mathcal{F} \vee \mathbb{S})$ as the completion of $A(S)^{\mathcal{F}}$ by its augmentation ideal. Recall that $\pi^{0}(\mathbb{B} S \vee \mathbb{S}) \cong \pi^{0}\left(B S_{+}\right) \cong A(S)_{I(S)}^{\wedge}($ see Remark 4.6. $)$

Theorem B. Let $\mathcal{F}$ be a saturated fusion system over a p-group $S$.

(1) The structure map $\sigma_{\mathcal{F}}$ induces an isomorphism $\pi^{0}(\mathbb{B} \mathcal{F} \vee \mathbb{S}) \cong\left(A(S)^{\mathcal{F}}\right)_{I}^{\wedge}$, where $I$ is the augmentation ideal of $A(S)^{\mathcal{F}}$.

(2) As abelian groups $\left(A(S)^{\mathcal{F}}\right)_{I}^{\wedge} \cong \mathbb{Z} \oplus\left(I \otimes \mathbb{Z}_{p}^{\wedge}\right)$ and $I \otimes \mathbb{Z}_{p}^{\wedge}$ is a free $\mathbb{Z}_{p}^{\wedge}$-module with one generator for every $\mathcal{F}$-conjugacy class of subgroups $H \leq S$ with $H \neq S$.

As the referee pointed out to us, it is probably more natural to consider $\mathbb{B} \mathcal{F}_{+}$ in Theorem $\mathrm{B}$ than $\mathbb{B} \mathcal{F} \vee \mathbb{S}$. However, in order to do this, one has to rewrite 
Ragnarsson's results in [9] and to redefine the structure map $\sigma_{\mathcal{F}}$ as a map $\mathbb{B} S_{+} \rightarrow$ $\mathbb{B} \mathcal{F}_{+}$rather than $\mathbb{B} S \rightarrow \mathbb{B} \mathcal{F}$. To avoid this and use Ragnarsson's work in its present form we chose to work with $\mathbb{B} \mathcal{F} \vee \mathbb{S}$.

Corollary C. If $(S, \mathcal{F}, \mathcal{L})$ is a p-local finite group then there is an isomorphism of rings $\pi^{0}\left(\left(|\mathcal{L}|_{p}^{\wedge}\right)_{+}\right) \cong\left(A(S)^{\mathcal{F}}\right)_{I}^{\wedge}$. In particular, if $G$ is a finite group then

$$
\pi^{0}\left(\left(B G_{p}^{\wedge}\right)_{+}\right) \cong\left(A(S)^{\mathcal{F}_{S}(G)}\right)_{I}^{\wedge} \cong \mathbb{Z} \oplus\left(\mathbb{Z}_{p}^{\wedge} \otimes I\right)
$$

where $I$ is the augmentation ideal of $A(S)^{\mathcal{F}_{S}(G)}$.

There is some overlap between this note and Ragnarsson's results in [8]. The authors would like to thank him for helpful discussions during a week long visit to Aberdeen in 2008.

We start in Section 2 with preliminaries about fusion systems, Burnside rings and completions. Then we devote Section 3 to prove Theorem $A$. Finally, in Section 4 we prove Theorem $B$ and in Proposition 4.12 we describe the spectrum of the prime ideals of $\mathbb{Z}_{(p)} \otimes\left(A(S)^{\mathcal{F}}\right)_{I}^{\wedge}$.

Notation: We denote the trivial subgroup of a groups $G$ by by $e$. If $X$ is a $G$-set and $H \leq G$, we write $X^{H}$ for the set of points of $X$ fixed by $H$ and $\left|X^{H}\right|$ for its cardinality. We will identify a $G$-set $X$ with its isomorphism class in the monoid $B(G)$. Throughout $\pi^{*}(-)$ will always mean stable cohomotopy groups of spectra or of pointed spaces. If $G$ is a finite group $\mathbb{B} G$ is the suspension spectrum of $B G$ with some basepoint chosen. If $X$ is any CW-complex (pointed or not) $X_{+}$ is obtained by adding a disjoint new basepoint to $X$. Finally, we reserve the letter $\mathbb{k}$ to denote a commutative ring, frequently it is torsion-free.

\section{Preliminaries: Fusion systems, Burnside Rings AND COMPletions}

Saturated fusion systems and their classifying spectrum. Let $S$ be a finite $p$-group. A fusion system over $S$ is a small category whose objects are the subgroups of $S$. The morphism sets $\mathcal{F}(P, Q)$ where $P, Q \leq S$ consists of group monomorphisms $P \rightarrow Q$ such that

(a) The set $\operatorname{Hom}_{S}(P, Q)$ of the morphisms $P \rightarrow Q$ obtained by conjugation in $S$, is contained in $\mathcal{F}(P, Q)$.

(b) Every morphism in $\mathcal{F}$ factors as an isomorphism in $\mathcal{F}$ followed by an inclusion of groups.

For example, if $S$ is a Sylow $p$-subgroup of a finite group $G$ then there results a fusion system $\mathcal{F}_{S}(G)$ where $\mathcal{F}(P, Q)=\operatorname{Hom}_{G}(P, Q)$ is the set of the homomorphisms

$c_{g}: P \stackrel{x \mapsto g x g^{-1}}{\longrightarrow} Q$ obtained by conjugation in $G$. This fusion system has a rigid structure which was first recognized and axiomatised by Puig and later by others. The set of axioms we will use are due to Broto-Levi-Oliver in [2].

Isomorphic objects in a fusion system $\mathcal{F}$ are called $\mathcal{F}$-conjugate and we will write $P \simeq_{\mathcal{F}} P^{\prime}$. A subgroup $P \leq S$ is called fully $\mathcal{F}$-centralised if $\left|C_{S}(P)\right| \geq\left|C_{S}\left(P^{\prime}\right)\right|$ for any $P^{\prime}$ which is $\mathcal{F}$-conjugate to $P$. Similarly, $P$ is called fully $\mathcal{F}$-normalised if $\left|N_{S}(P)\right| \geq\left|N_{S}\left(P^{\prime}\right)\right|$ for any $P^{\prime}$ which is $\mathcal{F}$-conjugate to $P$.

2.1. Definition. (See [2, Def. 1.2]) A fusion system $\mathcal{F}$ over $S$ is called saturated if

(I) Every fully $\mathcal{F}$-normalised $P \leq S$ is also fully $\mathcal{F}$-centralised and $\operatorname{Aut}_{S}(P)$ is a Sylow $p$-subgroup of $A u t_{\mathcal{F}}(P)$. 
(II) For every $P \leq S$ and every $\varphi \in \mathcal{F}(P, S)$ set

$$
N_{\varphi}=\left\{g \in N_{S} P: \varphi \circ c_{g} \circ \varphi^{-1} \in \operatorname{Aut}_{S}(\varphi(P))\right\} .
$$

If $\varphi(P)$ is fully $\mathcal{F}$-centralised then there is $\bar{\varphi} \in \mathcal{F}\left(N_{\varphi}, S\right)$ such that $\left.\bar{\varphi}\right|_{P}=\varphi$.

A subgroup $P \leq S$ is called $\mathcal{F}$-centric if $P$ and all its $\mathcal{F}$-conjugates contain their $S$-centraliser, see [2, Def. 1.6]. It is clear that if $P$ is $\mathcal{F}$-centric and $P \leq Q$ then $Q$ is also $\mathcal{F}$-centric. We will write $\mathcal{F}^{c}$ for the full subcategory of $\mathcal{F}$ generated by the $\mathcal{F}$-centric subgroups of $S$.

Ragnarsson constructed the classifying spectrum $\mathbb{B} \mathcal{F}$ in $[9$, Section 7]. It is equipped with a structure map $\sigma_{\mathcal{F}}: \mathbb{B} S \rightarrow \mathbb{B} \mathcal{F}$ and a transfer map $t_{\mathcal{F}}: \mathbb{B} \mathcal{F} \rightarrow \mathbb{B} S$. The fusion system is completely determined by its classifying spectrum $\mathbb{B} \mathcal{F}$ and the map $\sigma_{\mathcal{F}}$ in the sense that $\mathcal{F}$ can be recovered from this data by means of the set of stable maps $\{\mathbb{B} P, \mathbb{B} \mathcal{F}\}$ where $P \leq S$. The composite $t_{\mathcal{F}} \circ \sigma_{\mathcal{F}}$ is homotopic to the stable characteristic idempotent $\tilde{\omega}_{\mathcal{F}} \in\{\mathbb{B} S, \mathbb{B} S\}$ and $\sigma_{\mathcal{F}} \circ t_{\mathcal{F}} \simeq \operatorname{id}_{\mathbb{B} \mathcal{F}}$. In particular $\mathbb{B} \mathcal{F}$ splits off $\mathbb{B} S$.

The Burnside ring. The orbit category of a fusion system $\mathcal{F}$ is the small category $\mathcal{O}(\mathcal{F})$ whose objects are the subgroups of $S$ and $\mathcal{O}(\mathcal{F})(P, Q)=\mathcal{F}(P, Q) / \operatorname{Inn}(Q)$; See [2, Def. 2.1]. We write $\mathcal{O}\left(\mathcal{F}^{c}\right)$ for the full subcategory generated by the $\mathcal{F}$-centric subgroups of $S$.

In $[3, \S 4]$ we consider the category $\mathcal{O}\left(\mathcal{F}^{c}\right) \sqcup$ of the finite collections in $\mathcal{O}\left(\mathcal{F}^{c}\right)$. To make the construction precise one looks at the category of the contravariant functors $\mathcal{O}\left(\mathcal{F}^{c}\right) \rightarrow$ Sets which are isomorphic to $\coprod_{i=1}^{n} \mathcal{O}\left(\mathcal{F}^{c}\right)\left(-, P_{i}\right)$ where $n<\infty$ and $P_{i}$ are objects in $\mathcal{O}\left(\mathcal{F}^{c}\right)$. By construction, $\mathcal{O}\left(\mathcal{F}^{c}\right)_{\sqcup}$ is closed to finite coproducts and by Yoneda's Lemma it contains $\mathcal{O}\left(\mathcal{F}^{c}\right)$ as a full subcategory. The surprise is that $\mathcal{O}\left(\mathcal{F}^{c}\right)_{\sqcup}$ has finite products [7], [3, Theorem 1.2]. The product distributes over the coproduct and we define the Burnside ring $A(\mathcal{F})$ as a special case of the following general construction applied to $\mathcal{O}\left(\mathcal{F}^{c}\right) \sqcup$. See [5].

Consider an essentially small category $\mathcal{C}$ with finite coproducts and products and assume that the product distributes over the coproduct. Define $A(\mathcal{C})$ as the free abelian group generated by the isomorphism classes $[C]$ of the objects $C \in \mathcal{C}$ subject to the relation $\left[C_{1} \amalg C_{2}\right]=\left[C_{1}\right]+\left[C_{2}\right]$. The product in $\mathcal{C}$ gives $A(\mathcal{C})$ the structure of a ring where $\left[C_{1}\right] \cdot\left[C_{2}\right]=\left[C_{1} \times C_{2}\right]$. If the morphism sets of $\mathcal{C}$ are finite then for every isomorphism class $[C]$, the $\operatorname{ring} A(\mathcal{C})$ is equipped with a ring homomorphism

$$
\chi_{C}: A(\mathcal{C}) \rightarrow \mathbb{Z}, \quad(X \mapsto|\mathcal{C}(X, C)|) .
$$

For any commutative ring $\mathbb{k}$ we define $A_{\mathbb{k}}(\mathcal{C}):=\mathbb{k} \otimes_{\mathbb{Z}} A(\mathcal{C})$. We will denote

$$
A_{(p)}(\mathcal{C}):=A_{\mathbb{Z}_{(p)}}(\mathcal{C}) \quad \text { and } \quad A_{\hat{p}}(\mathcal{C}):=A_{\mathbb{Z}_{p}^{\wedge}}(\mathcal{C})
$$

If $C$ is an object of $\mathcal{C}$ we obtain, by tensoring with $\mathbb{k}$, another ring homomorphism

$$
\mathbb{k} \otimes \chi_{C}: A_{\mathbb{k}}(\mathcal{C}) \rightarrow \mathbb{k},
$$

that we denote also by $\chi_{C}$. It would be clear from the context which homomorphism we mean.

2.3. Example. The Burnside ring $A(G)$ of a finite group $G$ is $A$ (\{finite $G$-sets $\}$ ). It is a free group generated by the $G$-conjugacy classes of the transitive $G$-sets, each of which has as representative $G / H$ for some $H \leq G$. The homomorphism (2.2) for the subgroup $H \leq G$ assigns to any $G$-set $\mathrm{X}$ the cardinality of $X^{H}$, namely the subset of $X$ fixed by $H$. 
Given a prime $p$ we let $A(G ; p)$ be the subring of the finite $G$-sets whose isotropy groups are $p$-subgroups of $G$. This is the Burnside ring of the category of the finite $G$-sets whose isotropy groups are $p$-groups. From the homomorphisms (2.2), we obtain ring homomorphisms

$$
\chi: A(G) \rightarrow \prod_{\operatorname{ccs}(G)} \mathbb{k} \quad \text { and } \quad \chi: A(G ; p) \rightarrow \prod_{\operatorname{ccs}_{p}(G)} \mathbb{k},
$$

where $\operatorname{ccs}(G)$ and $\operatorname{ccs}_{p}(G)$ denote the set of conjugacy classes of the subgroups of $G$ and the set of the conjugacy classes of the $p$-subgroups of $G$. These homomorphisms are, in fact, ring monomorphisms provided $\mathbb{k}$ is torsion free. The trivial $G$-set $*$ is the identity element in $A(G)$ and more generally in $A_{\mathbb{k}}(G)$. We will use the integer $n$ to denote $n \cdot *$.

The augmentation homomorphism $\chi_{(1)}: A_{\mathbb{k}}(G) \rightarrow \mathbb{k}$ is of particular importance. It is usually denoted by $\epsilon$ and it sends a finite $G$-set $X$ to its cardinality $|X|$. Its kernel is the augmentation ideal $I_{\mathbb{k}}(G)$. If $\mathbb{k}$ is torsion free then $I_{\mathbb{k}}(G)$ has an additive basis $G / H-|G / H|$ with $H$ running over the $G$-conjugacy classes of subgroups $H$ of $G$ where $H \neq G$.

Going back to saturated fusion systems, we define $A(\mathcal{F})$ as $A\left(\mathcal{O}\left(\mathcal{F}^{c}\right) \sqcup\right)$. As an abelian group it is free with one basis element for each $\mathcal{F}$-conjugacy class of $\mathcal{F}$-centric subgroups of $S$ [3, Proposition 4.10]. We obtain a ring homomorphism

$$
\Phi: A(\mathcal{F}) \rightarrow \prod_{[P] \in \operatorname{ccs}\left(\mathcal{F}^{c}\right)} \mathbb{Z}, \quad[Q] \mapsto\left|\mathcal{O}\left(\mathcal{F}^{c}\right)(P, Q)\right|,
$$

where $\operatorname{ccs}\left(\mathcal{F}^{c}\right)$ stands for the set of the $\mathcal{F}$-conjugacy classes of the $\mathcal{F}$-centric subgroups of $S$. This is, in fact, a monomorphism by [3, Theorem 5.3] and therefore $\Phi_{(p)}=\mathbb{Z}_{(p)} \otimes \Phi$ is also a ring monomorphism.

In $\left[3\right.$, Theorem 5.4] we show that $\left(y_{P}\right)$ is in the image of $\Phi_{(p)}$ if and only if for any fully $\mathcal{F}$-normalised $Q \leq S$ the following congruence holds

$$
\sum_{P \in\left[\mathcal{F}^{c}\right]} n(Q, P) \cdot y_{P} \equiv 0 \bmod \left(\left|\operatorname{Out}_{S}(Q)\right|\right),
$$

where $P$ runs over a set of representatives for $\operatorname{ccs}\left(\mathcal{F}^{c}\right)$, where $\left(\left|\operatorname{Out}_{S}(Q)\right|\right)$ is the ideal in $\mathbb{Z}_{(p)}$ generated by $\left|\operatorname{Out}_{S}(Q)\right|$ and where

$$
n(Q, P)=\left|\left\{c_{s} \in \operatorname{Out}_{S}(Q):\langle s, Q\rangle \simeq_{\mathcal{F}} P\right\}\right| .
$$

Completion of rings. Let $I$ be an ideal in a commutative ring $R$. Then the ideals $I^{n}$ form a set of neighbourhoods for $0 \in R$ which generates the $I$-adic topology on $R$ and on any $R$-module $M$. The $I$-adic completion of an $R$-module $M$ is

$$
M_{I}^{\wedge}:=\lim _{n} M / I^{n} M
$$

It is clear from the construction that $M_{I}^{\wedge}$ is an $R_{I}^{\wedge}$-module. Also note that $M \rightarrow M_{I}^{\wedge}$ is injective if and only if the $I$-adic topology on $M$ is Hausdorff, namely $\cap_{n} I^{n} M=0$.

When $R$ is Noetherian one has more control on the $I$-completion. We recall below some basic results. Throughout, we write $\hat{M}$ for $M_{I}^{\wedge}$ and $\hat{R}=R_{I}^{\wedge}$ etc.

2.7. Theorem. ([4, Theorem 7.1].) If $R$ is Noetherian then $\hat{R}$ is Noetherian. Moreover, as an $R$-module, $\hat{R}$ is I-complete. 
2.8. Theorem. (Artin-Rees Lemma, [4, Theorem 7.2].) If $R$ is Noetherian and $M$ is a finitely generated $R$-module then $\hat{M}=\hat{R} \otimes_{R} M$. Moreover $\hat{R}$ is a flat $R$-module.

The $I$-adic topology on $R / I$ is discrete and therefore $\widehat{R / I}=R / I$. If $R$ is Noetherian then Theorem 2.8 gives rise to a short exact sequence $0 \rightarrow \hat{I} \rightarrow \hat{R} \rightarrow R / I \rightarrow 0$. 2.9. Theorem. ([4, Corollary 7.13].) If $R$ is Noetherian then $I \hat{R}=\hat{I}$. In particular $\hat{R}$ is $\hat{I}$-complete.

\section{3. $\mathcal{F}$-InVARIANT SETS}

In this section we prove Theorem A in the introduction. We begin studying and describing $\mathcal{F}$-invariant sets.

For any finite group $G$ denote by $B(G)$ the monoid, via disjoint unions, of the isomorphism classes of the finite $G$-sets, and by $A(G)=G r(B(G))$ its Grothendieck group, i.e. the Burnside ring of $G$. Recall that the product in this ring is induced by the product of $G$-sets. A homomorphism $\varphi: H \rightarrow G$ gives rise to a morphism of monoids $\varphi^{*}: B(G) \rightarrow B(H)$ and a homomorphism of rings $\varphi^{*}: A(G) \rightarrow A(H)$. If $\varphi$ is the inclusion of $H$ into $G$ we write $\operatorname{res}_{H}^{G}$ instead of $\varphi^{*}$.

Hence, if $\mathcal{F}$ is a saturated fusion system we have functors $B: \mathcal{F} \rightarrow$ Monoids and $A: \mathcal{F} \rightarrow$ Rings whose values on the subgroup $P$ is $B(P)$ and $A(P)$ respectively. Moreover, for any torsion-free commutative ring $\mathbb{k}$ we consider also the functor $A_{\mathrm{k}}=\mathbb{k} \otimes A$.

3.1. Definition. Let $\mathcal{F}$ be a saturated fusion system over $S$ and let $\mathbb{k}$ be a torsionfree commutative ring. We define the $\mathcal{F}$-invariant $S$-sets as $B(S)^{\mathcal{F}}=\varliminf_{\mathcal{F}} B$ and $A(S ; \mathcal{F}):=\operatorname{Gr}\left(B(S)^{\mathcal{F}}\right)$. We also define $A_{\mathbb{k}}(S ; \mathcal{F})=\mathbb{k} \otimes A(S ; \mathcal{F})$.

It is easy to see that $A(S ; \mathcal{F})$ is a subalgebra of $A(S)$ and therefore $A_{\mathbb{k}}(S ; \mathcal{F})$ is a $\mathbb{k}$-subalgebra of $A_{\mathbb{k}}(S)$ provided $\mathbb{k}$ is torsion-free.

3.2. Definition. Let $\mathcal{F}$ be a saturated fusion system over $S$ and let $\mathbb{k}$ be a torsionfree commutative ring. We define the ring of $\mathcal{F}$-invariant virtual $S$-sets as $A(S)^{\mathcal{F}}:=$ $\lim _{\mathcal{F}} A$. We also define $A_{\mathbb{k}}(S)^{\mathcal{F}}=\varliminf_{\lim _{\mathcal{F}}} A_{\mathbb{k}}$.

It is clear that $A_{\mathbb{k}}(S)^{\mathcal{F}}$ is a $\mathbb{k}$-subalgebra of $A_{\mathbb{k}}(S)$. The next goal is to prove that $A_{\mathbb{k}}(S ; \mathcal{F})=A_{\mathbb{k}}(S)^{\mathcal{F}}$ provided $\mathbb{k}$ is a torsion-free commutative ring.

By definition, an $S$-set $X$, which we identify with its isomorphism class in $B(S)$, is $\mathcal{F}$-invariant if for every $P \leq S$ and every $\varphi \in \mathcal{F}(P, S)$ the $P$-sets $\operatorname{res}_{P}^{S}(X)$ and $\varphi^{*}(X)$ are isomorphic. It is easy to see that $X$ is $\mathcal{F}$-invariant if and only if $\left|X^{P}\right|=\left|X^{P^{\prime}}\right|$ whenever $P$ and $P^{\prime}$ are $\mathcal{F}$-conjugate subgroups of $S$.

3.3. Proposition. For any fully $\mathcal{F}$-normalised $P \leq S$ there exists an $\mathcal{F}$-invariant S-set $\Omega_{P}$ such that

(1) All the isotropy groups of $\Omega_{P}$ are $\mathcal{F}$-conjugate to subgroups of $P$.

(2) $\Omega_{P}$ contains exactly one orbit isomorphic to $S / P$, and hence $\left|\left(\Omega_{P}\right)^{P}\right|=$ $\left|\frac{N_{S} P}{P}\right|$.

Proof. Let $(P)_{\mathcal{F}}$ denote the $\mathcal{F}$-conjugacy class of $P$. Let $P_{1}, \ldots, P_{k}$ be representatives for the $S$-conjugacy classes in $(P)_{\mathcal{F}}$. We may assume that $P_{1}=P$. Then $\left|N_{S} P_{1}\right| \geq\left|N_{S} P_{i}\right|$ for all $i$ and we consider the $S$-set

$$
\Omega_{0}=\coprod_{i} \frac{\left|N_{S} P_{1}\right|}{\left|N_{S} P_{i}\right|} \cdot S / P_{i} .
$$


Observe that for any $i$ we have

$$
\left|\Omega_{0}^{P_{i}}\right|=\frac{\left|N_{S} P_{1}\right|}{\left|N_{S} P_{i}\right|} \cdot\left|N_{S} P_{i} / P_{i}\right|=\frac{\left|N_{S} P\right|}{|P|} .
$$

Let $\mathcal{H}$ be the collection of all the subgroup $Q \leq S$ which are $\mathcal{F}$-conjugate to a proper subgroup of $P$. If $Q \notin \mathcal{H}$ then either $Q$ is not $\mathcal{F}$-conjugate to a subgroup of $P$ or it is $\mathcal{F}$-conjugate to $P$. In the first case it is clear that $\Omega_{0}^{Q}=\emptyset=\Omega_{0}^{Q^{\prime}}$ for any $Q^{\prime}$ which is $\mathcal{F}$-conjugate to $Q$. In the second case $Q$ is $S$-conjugate to one of the $P_{i}$ 's and therefore $\left|\Omega_{0}^{Q}\right|=\left|N_{S} P / P\right|$. We can now apply [2, Lemma 5.4] and deduce that there exists an $\mathcal{F}$-invariant $S$-set $\Omega$ which contains $\Omega_{0}$ and which also satisfies $\left|\Omega^{Q}\right|=\left|\Omega_{0}^{Q}\right|$ for all $Q \notin \mathcal{H}$. In particular it follows that all the isotropy groups of $\Omega$ belong to $\mathcal{H} \cup\left\{(P)_{\mathcal{F}}\right\}$ and that $\left|\Omega^{P}\right|=\left|N_{S} P / P\right|$.

Since $A_{\mathbb{k}}(S)^{\mathcal{F}}$ is a $\mathbb{k}$-subalgebra of $A_{\mathbb{k}}(S)$ the statement of the following lemma makes sense.

3.4. Lemma. For any $u \in A_{\mathbb{k}}(S)^{\mathcal{F}}$ and any two $\mathcal{F}$-conjugate subgroups $Q, Q^{\prime} \leq S$, $\chi_{Q}(u)=\chi_{Q^{\prime}}(u)$ where $\chi_{Q}$ is defined in (2.2) (see also (2.4)).

Proof. Note that $\chi_{Q}(u)$ is the coefficient of the trivial $Q$-set $Q / Q$ in $\operatorname{res}_{Q}^{S}(u) \in$ $A_{\mathbb{k}}(Q)$. Similarly $\chi_{Q^{\prime}}(u)$ is the coefficient of the trivial $Q^{\prime}$-set in $\operatorname{res}_{Q^{\prime}}^{S}(u)$. In turn, this is the coefficient of the trivial $Q$-set $Q / Q$ in $\varphi^{*}(u) \in A_{\mathbb{k}}(Q)$ where $\varphi \in \mathcal{F}(Q, S)$ is an isomorphism of $Q$ onto $Q^{\prime}$. The result follows since $u \in A_{\mathbb{k}}(S)^{\mathcal{F}}$ whence $\operatorname{res}_{Q}^{S}(u)=\varphi^{*}(u)$.

3.5. Remark. Consider the restriction of (2.4) to $\chi: A_{\mathbb{k}}(S)^{\mathcal{F}} \rightarrow \prod_{\operatorname{ccs}(S)} \mathbb{k}$. From Lemma 3.4 it is clear that $\chi$ factors through an injective ring homomorphism $\chi: A_{\mathbb{k}}(S)^{\mathcal{F}} \rightarrow \prod_{\operatorname{ccs}(\mathcal{F})} \mathbb{k}$, where the product runs through the $\mathcal{F}$-conjugacy classes of the subgroups of $S$.

If $X$ is an $\mathcal{F}$-invariant $S$-set then it represents an element in $A_{\mathbb{k}}(S)^{\mathcal{F}}$. Thus, $A_{\mathbb{k}}(S ; \mathcal{F}) \subseteq A_{\mathbb{k}}(S)^{\mathcal{F}}$, where we regard both as $\mathbb{k}$-subalgebras of $A_{\mathbb{k}}(S)$. Now we are ready to prove that this inclusion is an equality and to describe a basis for $A_{\mathbb{k}}(S ; \mathcal{F})$.

3.6. Proposition. Let $\mathcal{F}$ be a saturated fusion system over $S$ and let $\mathbb{k}$ be a torsionfree commutative ring. Let $Q_{1}, \ldots, Q_{r}$ be representatives for the $\mathcal{F}$-conjugacy classes of the subgroups of $S$ such that the $Q_{i}$ 's are fully $\mathcal{F}$-normalised. Then

(1) $A_{\mathbb{k}}(S ; \mathcal{F})=A_{\mathbb{k}}(S)^{\mathcal{F}}$.

(2) $A_{\mathbb{k}}(S ; \mathcal{F})$ is a free $\mathbb{k}$-module with basis $\Omega_{Q_{1}}, \ldots, \Omega_{Q_{r}}$ where $\Omega_{Q_{i}}$ are defined in Proposition 3.3.

Proof. We arrange the $Q_{i}$ 's so that $\left|Q_{i}\right| \geq\left|Q_{i+1}\right|$. Thus, if $j>i$ then $Q_{i}$ is not $\mathcal{F}$-conjugate to a subgroup of $Q_{j}$ and in particular $(S / P)^{Q_{i}}=\emptyset$ if $P$ is $\mathcal{F}$-conjugate to $Q_{j}$. From Remark 3.5 and Example 2.3, there is a ring monomorphism

$$
\chi: A_{\mathbb{k}}(S)^{\mathcal{F}} \rightarrow \prod_{i=1}^{r} \mathbb{k}, \quad \chi=\left(\chi_{Q_{1}}, \ldots, \chi_{Q_{r}}\right) .
$$

Since $\left(\Omega_{Q_{i}}\right)^{Q_{j}}=\emptyset$ if $j<i$ we see from Proposition 3.3 that

$$
\chi\left(\Omega_{Q_{i}}\right)=(\underbrace{0, \ldots, 0}_{i-1 \text { times }}, \frac{\left|N_{S}\left(Q_{i}\right)\right|}{\left|Q_{i}\right|}, \ldots)
$$


where $\frac{\left|N_{S}\left(Q_{i}\right)\right|}{\left|Q_{i}\right|} \neq 0$ because $\mathbb{k}$ is torsion-free. Thus, $\chi\left(\Omega_{Q_{1}}\right), \ldots, \chi\left(\Omega_{Q_{r}}\right)$ are $\mathbb{k}$ linearly independent in $\mathbb{k}^{r}$ and therefore $\Omega_{Q_{1}}, \ldots, \Omega_{Q_{r}}$ form a basis for the $\mathbb{k}$ submodule $B \leq A_{\mathbb{k}}(S)$ it generates. We now have

$$
B \leq A_{\mathbb{k}}(S ; \mathcal{F}) \leq A_{\mathbb{k}}(S)^{\mathcal{F}} \stackrel{\chi}{\longmapsto} \prod_{j=1}^{r} \mathbb{k} .
$$

It remains to prove that $B=A_{\mathbb{k}}(S)^{\mathcal{F}}$.

Assume to the contrary that this is not the case and let $m$ be the maximal integer with the property that there exists some $u \in A_{\mathbb{k}}(S)^{\mathcal{F}} \backslash B$ such that $\chi_{Q_{i}}(u)=0$ for all $i=1, \ldots, m-1$. Clearly $m \leq r$ or else $u=0$ because $\chi$ is injective. Also $\chi_{Q_{m}}(u) \neq 0$. Express $u$ in terms of the basis of $A_{\mathbb{k}}(S)$, namely

$$
u=\sum_{(Q) \in \operatorname{ccs}(S)} \alpha_{Q} \cdot S / Q
$$

and consider the smallest integer $j=1, \ldots, r$ for which there is some $R \simeq_{\mathcal{F}} Q_{j}$ such that $\alpha_{R} \neq 0$. Thus, if $\alpha_{Q} \neq 0$ then $|R| \geq|Q|$. Since $\mathbb{k}$ is torsion-free, by Lemma 3.4 and Proposition 3.3 we have

$$
\chi_{Q_{j}}(u)=\chi_{R}(u)=\sum_{(Q) \in \operatorname{ccs}(S)} \alpha_{Q} \cdot \chi_{R}(S / Q)=\alpha_{R} \cdot\left|N_{S} R / R\right| \neq 0 .
$$

It follows from the definition of $m$ that $j \geq m$. Now, if $\alpha_{Q} \neq 0$ then $Q$ is $\mathcal{F}$ conjugate to $Q_{i}$ where $i \geq j$, and therefore $i \geq m$. We deduce that $\left|Q_{m}\right| \geq|Q|$ so $(S / Q)^{Q_{m}}=\emptyset$ unless $Q$ is $S$-conjugate to $Q_{m}$. It follows that

$$
\chi_{Q_{m}}(u)=\alpha_{Q_{m}} \cdot\left|N_{S} Q_{m} / Q_{m}\right|
$$

which implies that $\alpha_{Q_{m}} \neq 0$ because $\chi_{Q_{m}}(u) \neq 0$. From the minimality of $j$ we deduce that $j \leq m$ and therefore $j=m$.

Consider $v:=u-\alpha_{Q_{m}} \Omega_{Q_{m}}$. Clearly $v \in A_{\mathbb{k}}(S)^{\mathcal{F}} \backslash B$. Moreover, if $i<m$ then $\left(\Omega_{Q_{m}}\right)^{Q_{i}}=\emptyset$ by Proposition 3.3 so

$$
\chi_{Q_{i}}(v)=\chi_{Q_{i}}(u)-\chi_{Q_{i}}\left(\alpha_{Q_{m}} \Omega_{Q_{m}}\right)=0, \quad \text { if } i<m .
$$

From (3.7) we also deduce that

$$
\chi_{Q_{m}}(v)=\chi_{Q_{m}}(u)-\chi_{Q_{m}}\left(\alpha_{Q_{m}} \Omega_{Q_{m}}\right)=\chi_{Q_{m}}(u)-\alpha_{Q_{m}}\left|\frac{N_{S} Q_{m}}{Q_{m}}\right|=0 .
$$

This contradicts the maximality of $m$ and therefore $B=A_{\mathbb{k}}(S)^{\mathcal{F}}$.

3.8. Remark. It follows from Proposition 3.6 that if $\mathbb{k}$ is torsion free then

$$
A_{\mathbb{k}}(S ; \mathcal{F})=\mathbb{k} \otimes A(S ; \mathcal{F})=\mathbb{k} \otimes A(S)^{\mathcal{F}}=A_{\mathbb{k}}(S)^{\mathcal{F}} \leq A_{\mathbb{k}}(S) .
$$

3.9. Example. Let $S$ be a Sylow $p$-subgroup of a finite group $G$. If $X$ is a finite $G$-set then $\operatorname{res}_{S}^{G}(X)$ is an $\mathcal{F}_{S}(G)$-invariant $S$-set. This gives a ring homomorphism res: $A(G ; p) \rightarrow A(S ; \mathcal{F})$, see Example 2.3. Observe that a basis for $A(G ; p)$ is the set $\left\{G / Q_{i}\right\}_{i=1}^{r}$ and a basis for $A\left(S ; \mathcal{F}_{S}(G)\right)$ is the set $\left\{\Omega_{Q_{i}}\right\}_{i=1}^{r}$ where $Q_{i} \leq S$ are chosen as in Proposition 3.6. If $P=Q_{i}$ then

$$
\operatorname{res}_{S}^{G}(G / P)=\sum_{g \in S \backslash G / P} S / S \cap{ }^{g} P=\left|\frac{N_{G} P}{N_{S} P}\right| \cdot S / P+Y
$$

where $Y$ is a finite $S$-set whose isotropy groups are conjugate in $G$ to subgroups $Q \leq P$ and $Q$ is not $S$-conjugate to $P$ itself. Thus, by Propositions 3.3 and 3.6, 
the matrix which represents res: $A(G ; p) \rightarrow A(S ; \mathcal{F})$ with respect to the bases described above, is upper triangular with diagonal entries $\left|\frac{N_{G} Q_{i}}{N_{S} Q_{i}}\right|$. Since $N_{S}\left(Q_{i}\right)$ is a Sylow $p$-subgroup of $N_{G} Q_{i}$, this matrix is invertible in $\mathbb{Z}_{(p)}$. Hence $\mathbb{Z}_{(p)} \otimes$ res is an isomorphism $A_{(p)}(S ; \mathcal{F}) \cong A_{(p)}(G ; p)$.

Proof of Theorem A. In light of Proposition 3.6 and remark 3.8, we may replace $A_{(p)}(S)^{\mathcal{F}}$ with $A_{(p)}(S ; \mathcal{F})$. Let $Q_{1}, Q_{1}, \ldots, Q_{r}$ be fully $\mathcal{F}$-normalised representatives for the $\mathcal{F}$-conjugacy classes of the subgroups of $S$. We order them in such a way that $Q_{1}, \ldots, Q_{s}$ are $\mathcal{F}$-centric, $Q_{s+1}, \ldots, Q_{r}$ are not $\mathcal{F}$-centric and $\left|Q_{1}\right| \geq \cdots \geq\left|Q_{s}\right|$. In Proposition 3.6 we showed that $A_{(p)}(S ; \mathcal{F})$ is a free $\mathbb{Z}_{(p)}$-module generated by the $S$-sets $\Omega_{Q_{i}}$.

Consider the restriction $\chi: A_{(p)}(S ; \mathcal{F}) \rightarrow \prod_{j=1}^{r} \mathbb{Z}_{(p)}$ of the ring homomorphism (2.4) for $\mathbb{k}=\mathbb{Z}_{(p)}$. See also Remark 3.5. Then by Proposition 3.3, $\chi_{Q_{j}}\left(\Omega_{Q_{i}}\right)=0$ if $j<i$ and $\chi_{Q_{i}}\left(\Omega_{Q_{i}}\right)=\left|\frac{N_{S} Q_{i}}{Q_{i}}\right| \neq 0$. From this it easily follows that $N$ is the kernel of the composite homomorphism

$$
A_{(p)}(S ; \mathcal{F}) \stackrel{\chi}{\longrightarrow} \prod_{\operatorname{ccs}(\mathcal{F})} \mathbb{Z}_{(p)} \stackrel{\operatorname{proj}}{\longrightarrow} \prod_{\operatorname{ccs}\left(\mathcal{F}^{c}\right)} \mathbb{Z}_{(p)}
$$

because every $u \in N$ must be a linear combination of those $\Omega_{Q_{i}}$ 's such that $Q_{i}$ is not $\mathcal{F}$-centric. Here, $\operatorname{ccs}(\mathcal{F})$ is the set of the $\mathcal{F}$ - conjugacy classes of the subgroups of $S$ and $\operatorname{ccs}\left(\mathcal{F}^{c}\right)$ is the set of the $\mathcal{F}$-conjugacy classes of the $\mathcal{F}$-centric subgroups of $S$. We deduce that there is a ring monomorphism

$$
\bar{\chi}: A_{(p)}(S ; \mathcal{F}) / N \rightarrow \prod_{\operatorname{ccs}\left(\mathcal{F}^{c}\right)} \mathbb{Z}_{(p)}, \quad \bar{\chi}=\left(\chi_{Q_{1}}, \ldots, \chi_{Q_{s}}\right) .
$$

In light of $(2.5)$, it remains to show that $\operatorname{Im}(\bar{\chi})=\operatorname{Im}\left(\Phi_{(p)}\right)$. First we claim that $\operatorname{Im}(\bar{\chi}) \subseteq \operatorname{Im}\left(\Phi_{(p)}\right)$. To see this we choose some $\mathcal{F}$-invariant $S$-set $X$. Then for every $i \leq s$ we have $\bar{\chi}_{Q_{i}}(X)=\left|X^{Q_{i}}\right|$ and to see that $\bar{\chi}(X)$ belongs to $\operatorname{Im}\left(\Phi_{(p)}\right)$ we use (2.6). By Cauchy-Frobenius formula, for any fully $\mathcal{F}$-normalised $Q \in \mathcal{F}^{c}$,

$$
\begin{aligned}
& \sum_{P \in \operatorname{ccs}\left(\mathcal{F}^{c}\right)} n(Q, P) \cdot\left|X^{P}\right|=\sum_{P \in \operatorname{ccs}\left(\mathcal{F}^{c}\right)}\left(\sum_{s \in \frac{N_{S} Q}{Q},\langle Q, s\rangle \simeq_{\mathcal{F}} P}\left|X^{P}\right|\right)= \\
& \sum_{s \in \frac{N_{S} Q}{Q}}\left|\left(X^{Q}\right)^{s}\right|=\left|\frac{N_{S} Q}{Q}\right| \cdot \#\left\{\text { orbits of } \frac{N_{S} Q}{Q} \text { on } X^{Q}\right\}=0 \bmod \left(\left|\frac{N_{S} Q}{Q}\right|\right) .
\end{aligned}
$$

Since $Q$ is $\mathcal{F}$-centric and fully $\mathcal{F}$-normalised, $\left|\operatorname{Out}_{S}(Q)\right|=\left|\frac{N_{S} Q}{Q}\right|$, hence $\bar{\chi}(X) \in$ $\operatorname{Im}\left(\Phi_{(p)}\right)$.

Now, a basis for $\operatorname{Im}(\bar{\chi})$ is the set $\left\{\bar{\chi}\left(\Omega_{Q_{i}}\right)\right\}_{i=1}^{s}$ and a basis for $\operatorname{Im}\left(\Phi_{(p)}\right)$ is the set $\left\{\Phi_{(p)}\left(\left[Q_{i}\right]\right)\right\}_{i=1}^{s}$. Note that for every $i$ we have $\bar{\chi}_{Q_{j}}\left(\Omega_{i}\right)=\Phi_{Q_{j}}\left(\left[Q_{i}\right]\right)=0$ if $j<i$ by Proposition 3.3 and (2.5). Furthermore, $\Phi_{Q_{i}}\left(\left[Q_{i}\right]\right)=\left|\operatorname{Out}_{\mathcal{F}}\left(Q_{i}\right)\right|$ and $\bar{\chi}_{Q_{i}}\left(\Omega_{Q_{i}}\right)=$ $\left|\frac{N_{S} Q_{i}}{Q_{i}}\right|$ differ by a unit in $\mathbb{Z}_{(p)}$ because $\operatorname{Out}_{S}\left(Q_{i}\right)$ is a Sylow $p$-subgroup of $\operatorname{Out}_{\mathcal{F}}\left(Q_{i}\right)$. It is now an easy exercise in linear algebra to see that $\operatorname{Im}(\bar{\chi})=\operatorname{Im}\left(\Phi_{(p)}\right)$.

\section{Segal's CONJECture}

The aim of this section is to prove Theorem B of the introduction. We start describing the augmentation ideal $I_{\mathbb{k}}(S ; \mathcal{F})$ of $A_{\mathbb{k}}(S ; \mathcal{F})$ and modules for which the $I_{\mathbb{k}}(S ; \mathcal{F})$-adic topology is equivalent to the $p$-adic topology. To avoid triviality we will assume throughout this section that the saturated fusion system $\mathcal{F}$ are 
defined over a non trivial $p$-group $S \neq 1$. We recall from Proposition 3.6 that $A_{\mathbb{k}}(S ; \mathcal{F})=A_{\mathbb{k}}(S)^{\mathcal{F}}$ if $\mathbb{k}$ is torsion-free.

Recall from Remark 3.8 that if $\mathbb{k}$ is torsion-free then $A_{\mathbb{k}}(S ; \mathcal{F})$ is a $\mathbb{k}$-subalgebra of $A_{\mathbb{k}}(S)$.

4.1. Definition. There is a standard augmentation map $\epsilon: A_{\mathbb{k}}(S) \rightarrow \mathbb{k}$ sending an $S$-set $X$ to its cardinality. Let $I_{\mathbb{k}}(S ; \mathcal{F})$ denote the kernel of $\epsilon: A_{\mathbb{k}}(S ; \mathcal{F}) \rightarrow \mathbb{k}$.

Recall that the trivial $S$-set is the unit in $A_{\mathbb{k}}(S)$. We will therefore use integers to represent the corresponding elements in $A_{\mathrm{k}}(S)$.

4.2. Lemma. With the notation and hypotheses of Proposition 3.6, $I_{\mathbb{k}}(S ; \mathcal{F})$ is a free $\mathbb{k}$-module of rank $r-1$ with basis $\left\{\Omega_{Q_{i}}-\left|\Omega_{Q_{i}}\right|\right\}$ where $i$ runs through the indices such that $Q_{i} \neq S$.

Proof. We may assume that $Q_{1}=S$ and that $\Omega_{Q_{1}}=*$. It is clear from Proposition 3.6 that $\Omega_{Q_{2}}-\left|\Omega_{Q_{2}}\right|, \ldots, \Omega_{Q_{r}}-\left|\Omega_{Q_{r}}\right|$ are $\mathbb{k}$-linearly independent in $A_{\mathbb{k}}(S ; \mathcal{F})$. The result follows from the fact that the $\Omega_{Q_{i}}$ 's form a basis for $A_{\mathbb{k}}(S ; \mathcal{F})$.

4.3. Proposition. Assume that $\mathbb{k}$ is torsion-free. Then the $I_{\mathbb{k}}(S ; \mathcal{F})$-adic topology on $I_{\mathrm{k}}(S ; \mathcal{F})$ is equivalent to the $p$-adic topology.

Proof. May and McClure show in $\left[6\right.$, p. 212] that $I(S)^{n+1} \subseteq p \cdot I(S)$ where $|S|=p^{n}$. Therefore, after tensoring with $\mathbb{k}$ and using Lemma 4.2, we see that $I_{\mathbb{k}}(S)^{n+1} \subseteq$ $p \cdot I_{\mathrm{k}}(S)$. In addition,

$$
I_{\mathbb{k}}(S ; \mathcal{F})^{n+1} \subseteq A_{\mathbb{k}}(S ; \mathcal{F}) \cap I_{\mathbb{k}}(S)^{n+1} \subseteq A_{\mathbb{k}}(S ; \mathcal{F}) \cap p \cdot I_{\mathbb{k}}(S)=p \cdot I_{\mathbb{k}}(S ; \mathcal{F}),
$$

where for the last equality we argue as follows. First, the inclusion $\supseteq$ is obvious. Consider $u \in A_{\mathbb{k}}(S ; \mathcal{F}) \cap p \cdot I_{\mathbb{k}}(S)$ and use Proposition 3.6 to write $u=\sum_{i=1}^{r} \beta_{i} \Omega_{Q_{i}}$. Clearly, $u \in p \cdot A_{\mathbb{k}}(S)$ and by looking at the coefficients of $S / Q_{i}$, one easily deduces from Proposition 3.3 that $\beta_{i}=p \cdot \alpha_{i}$ for all $i$. It follows that $u=p \cdot\left(\sum_{i} \alpha_{i} \Omega_{Q_{i}}\right)$. Now, $\epsilon(u)=0$ and since $\mathbb{k}$ is torsion free, we deduce that $\epsilon\left(\sum_{i} \alpha_{i} \Omega_{Q_{i}}\right)=0$. Therefore $u \in p \cdot I_{\mathbb{k}}(S ; \mathcal{F})$.

We now claim that

$$
p^{n} \cdot I_{\mathbb{k}}(S ; \mathcal{F}) \subseteq I_{\mathbb{k}}(S ; \mathcal{F})^{2} .
$$

To see this consider $\eta=(S / e-|S|)$ as an element in $I_{\mathbb{k}}(S ; \mathcal{F})$. Observe that $\{(S / Q-|S / Q|)\}$ form a basis for $I_{\mathbb{k}}(S)$, where $Q$ runs through the representatives for the $S$-conjugacy classes of the subgroups of $S$ different from $S$. By inspection

$$
\eta \cdot(S / Q-|S / Q|)=-|S| \cdot(S / Q-|S / Q|)=\left(-p^{n}\right) \cdot(S / Q-|S / Q|) .
$$

That is, multiplication by $\eta$ results in multiplication by $\left(-p^{n}\right)$ in $I_{\mathbb{k}}(S)$. Therefore

$$
I_{\mathbb{k}}(S ; \mathcal{F})^{2} \supseteq \eta \cdot I_{\mathbb{k}}(S ; \mathcal{F})=p^{n} \cdot I_{\mathbb{k}}(S ; \mathcal{F}) .
$$

This completes the proof.

4.4. Corollary. If $\mathbb{k}$ is torsion-free then $I_{\mathbb{k}}(S ; \mathcal{F})_{I_{\mathbb{k}}(S ; \mathcal{F})}^{\wedge}=I_{\mathbb{k}}(S ; \mathcal{F})_{p}^{\wedge}=I_{\mathbb{k}_{p}}(S ; \mathcal{F})$.

Proof. The first equality follows from Proposition 4.3, the second follows since $I_{\mathrm{k}}(S ; \mathcal{F})$ is a finitely generated free $\mathbb{k}$-module by Lemma 4.2 and therefore $I_{\mathrm{k}}(S ; \mathcal{F})_{p}^{\wedge}=$ $\mathbb{k}_{p}^{\wedge} \otimes_{\mathbb{k}} I_{\mathbb{k}}(S ; \mathcal{F})$. 
Any group homomorphism $\varphi: H \rightarrow G$ induces a ring homomorphism $\varphi^{*}: A_{\mathrm{k}}(G) \rightarrow$ $A_{\mathrm{k}}(H)$. Note that $\varphi^{*}$ carries $I_{\mathrm{k}}(G)$ into $I_{\mathrm{k}}(H)$ and hence there results a natural ring homomorphism $\varphi^{*}: A_{\mathbb{k}}(G)_{I_{\mathrm{k}}(G)}^{\wedge} \rightarrow A_{\mathbb{k}}(H)_{I_{\mathrm{k}}(H)}^{\wedge}$. Thus there is a contravariant functor $\widehat{A_{\mathrm{k}}}: \mathcal{F} \rightarrow$ Rings which maps $P \leq S$ to $\widehat{A_{\mathrm{k}}}(P)=A_{\mathrm{k}}(P)_{I_{\mathrm{k}}(P)}^{\wedge}$.

4.5. Definition. Let $\mathcal{F}$ be a saturated fusion system over the $p$-group $S$. We define the ring of $\mathcal{F}$-invariant completed $S$-sets as $\widehat{A_{\mathbb{k}}}(S)^{\mathcal{F}}=\varliminf_{\mathcal{F}} \widehat{A_{\mathbb{k}}}$.

Notice that $\widehat{A_{\mathrm{k}}}(S)^{\mathcal{F}}$ is the subring of $\widehat{A_{\mathrm{k}}}(S)$ of the elements $\hat{u}$ such that $\varphi^{*}(\hat{u})=$ $\operatorname{res}_{P}^{S}(\hat{u})$ for all $\varphi \in \mathcal{F}(P, S)$ and any subgroup $P \leq S$.

4.6. Remark. Notice that for any pointed CW-complex $X$ there is a natural equivalence $\Sigma^{\infty} X \vee \mathbb{S} \simeq \Sigma^{\infty}\left(X_{+}\right)$in the homotopy category of spectra (see [1].)

Recall from $\S 2$ that the classifying spectrum of a saturated fusion system $\mathcal{F}$ over $S$ is equipped with a structure map $\sigma_{\mathcal{F}}: \mathbb{B} S \rightarrow \mathbb{B} \mathcal{F}$ where $\mathbb{B} S$ is the suspension spectrum of $B S$ with some chosen basepoint. From the remark above we see that for any spectrum $E$ there is are isomorphisms $\{\mathbb{B} S \vee \mathbb{S}, E\}=\left\{\Sigma^{\infty} B S_{+}, E\right\}=E^{0}\left(B S_{+}\right)$. In particular, for $E=\mathbb{S}$ we have $\pi^{0}(\mathbb{B} S \vee \mathbb{S}) \cong \pi^{0}\left(B S_{+}\right)$.

4.7. Lemma. For any saturated fusion system $\mathcal{F}$ over $S$ the map $\sigma_{\mathcal{F}} \vee \mathbb{S}$ induces a split monomorphism

$$
\pi^{0}(\mathbb{B} \mathcal{F} \vee \mathbb{S}) \stackrel{\left(\sigma_{\mathcal{F}} \vee \mathbb{S}\right)^{*}}{\longrightarrow} \pi^{0}(\mathbb{B} S \vee \mathbb{S}) \cong \pi^{0}\left(B S_{+}\right) \cong \widehat{A}(S)
$$

whose image is isomorphic to the subring $\widehat{A}(S)^{\mathcal{F}}$.

Proof. Consider the transfer map $t_{\mathcal{F}}: \mathbb{B} \mathcal{F} \rightarrow \mathbb{B} S$, see $\S 2$ and [9]. The composite $\sigma_{\mathcal{F}} \circ t_{\mathcal{F}}$ is homotopic to id $\mathbb{B F}_{\mathcal{F}}$ so $\pi^{0}\left(t_{\mathcal{F}} \vee \mathbb{S}\right)$ is a left inverse for $\pi^{0}\left(\sigma_{\mathcal{F}} \vee \mathbb{S}\right)$ which is therefore a split monomorphism. Set $\tilde{\omega}_{\mathcal{F}}=t_{\mathcal{F}} \circ \sigma_{\mathcal{F}}$, see [9].

For any spectrum $E$, the image of $E^{0}(\mathbb{B} \mathcal{F}) \stackrel{E^{0}\left(\sigma_{\mathcal{F}}\right)}{\longrightarrow} E^{0}(\mathbb{B} S)$ is equal to the image of $E^{0}(\mathbb{B} S) \stackrel{E^{0}\left(\tilde{\omega}_{\mathcal{F}}\right)}{\longrightarrow} E^{0}(\mathbb{B} S)$. By [9, Corollary 6.4], the image of $E^{0}\left(\tilde{\omega}_{\mathcal{F}}\right)$ is equal to the set of homotopy classes $f \in\{\mathbb{B} S, E\}$ which are $\mathcal{F}$-invariant, that is

$$
\{\mathbb{B F}, E\} \stackrel{E^{0}\left(\sigma_{\mathcal{F}}\right)}{\cong} \lim _{P \in \mathcal{F} \text { op }}\{\mathbb{B} P, E\}=E^{0}(\mathbb{B} S)^{\mathcal{F}}
$$

where $\mathbb{B}: P \mapsto \mathbb{B} P$ is a functor from $\mathcal{F}$ to the category of spectra. It follows that

$$
E^{0}(\mathbb{B} \mathcal{F} \vee \mathbb{S}) \stackrel{E^{0}\left(\sigma_{\mathcal{F}} \vee \mathbb{S}\right)}{\cong} \lim _{P \in \mathcal{F} \text { op }} E^{0}(\mathbb{B} P \vee \mathbb{S})=E^{0}(\mathbb{B} S \vee \mathbb{S})^{\mathcal{F}}
$$

The assignments below induce isomorphic functors $\mathcal{F}^{\mathrm{op}} \rightarrow \mathbf{A b}$,

$$
P \mapsto\{\mathbb{B} P \vee \mathbb{S}, E\} \quad \text { and } \quad P \mapsto\left\{\Sigma^{\infty} B P_{+}, E\right\}=E^{0}\left(B P_{+}\right) .
$$

This is because we have cofibre sequences $\mathbb{S} \rightarrow \Sigma^{\infty} B P_{+} \rightarrow \mathbb{B} P$ and $\mathbb{S} \rightarrow \mathbb{B} P \vee \mathbb{S} \rightarrow$ $\mathbb{B} P$ in which $\mathbb{S}$ is a retract. Thus, $\{\mathbb{B} S \vee \mathbb{S}, E\}^{\mathcal{F}} \cong E^{0}\left(B S_{+}\right)^{\mathcal{F}}$. We now specialise to $E=\mathbb{S}$. Using the fact that the isomorphism $\pi^{0}\left(B G_{+}\right) \cong \widehat{A}(G)$ is natural in the group $G$, we obtain a natural isomorphism of rings $\pi^{0}\left(B S_{+}\right)^{\mathcal{F}} \cong \widehat{A}(S)^{\mathcal{F}}$ as a subring of $\pi^{0}(\mathbb{B} S \vee \mathbb{S}) \cong \pi^{0}\left(B S_{+}\right) \cong \hat{A}(S)$. The result now follows from (4.8).

In order to simplify the notation, we will denote the $I_{\mathbb{k}}(S ; \mathcal{F})$-adic completion of $A_{\mathbb{k}}(S ; \mathcal{F})$ by $\widehat{A_{\mathbb{k}}}(S ; \mathcal{F})$. Similarly $\widehat{I_{\mathbb{k}}}(S ; \mathcal{F})$ is the $I_{\mathbb{k}}(S ; \mathcal{F})$-adic completion of 
$I_{\mathbb{k}}(S ; \mathcal{F})$. When $\mathcal{F}$ is the trivial fusion system over $S$ this clearly becomes $\widehat{A_{\mathbb{k}}}(S)$ and $\widehat{I_{\mathbb{k}}}(S)$. Set $\widehat{I_{\mathbb{k}}}(S)^{\mathcal{F}}=\widehat{I_{\mathbb{k}}}(S) \cap \widehat{A_{\mathbb{k}}}(S)^{\mathcal{F}}$.

4.9. Proposition. Let $\mathbb{k}$ be a torsion-free commutative ring such that $\mathbb{k}_{p}^{\wedge}$ is also torsion-free. Assume that $\mathcal{F}$ is a saturated fusion system over $S \neq 1$. Then

(1) $\widehat{I}_{\mathbb{k}}(S)^{\mathcal{F}} \cong \widehat{I}_{\mathbb{k}}(S ; \mathcal{F}) \cong \mathbb{k}_{p}^{\wedge} \otimes_{\mathbb{k}} I_{\mathbb{k}}(S ; \mathcal{F})$.

(2) $\widehat{A_{\mathrm{k}}}(S)^{\mathcal{F}} \cong \widehat{A_{\mathrm{k}}}(S ; \mathcal{F})$.

(3) $\widehat{A_{\mathbb{k}}}(S ; \mathcal{F})$ contains $I_{\mathbb{k}_{p}}(S ; \mathcal{F})$ as an ideal with quotient $\mathbb{k}$. If $\mathbb{k}$ is $p$-complete then $\widehat{A_{\mathbb{k}}}(S ; \mathcal{F}) \cong A_{\mathbb{k}}(S ; \mathcal{F})$.

Proof. We first observe that if $M$ is an $R$-module and $I$ is an ideal in $R$ then the $I$-adic topology on $M / I M$ is discrete and the short exact sequences $0 \rightarrow \frac{I M}{I^{n} M} \rightarrow$ $\frac{M}{I^{n} M} \rightarrow \frac{M}{I M} \rightarrow 0$ for all $n \geq 1$ yield a short exact sequence

$$
0 \rightarrow(I M)_{I}^{\wedge} \rightarrow M_{I}^{\wedge} \rightarrow M / I M \rightarrow 0
$$

because the tower $\left\{I M / I^{n} M\right\}_{n \geq 0}$ is Mittag-Leffler. In particular, the augmentation $A_{\mathbb{k}}(S) \stackrel{\epsilon}{\rightarrow} \mathbb{k}$ extends to a short exact sequence

$$
0 \rightarrow \widehat{I_{\mathbb{k}}}(S ; \mathcal{F}) \rightarrow \widehat{A_{\mathrm{k}}}(S ; \mathcal{F}) \stackrel{\hat{\epsilon}}{\rightarrow} \mathbb{k} \rightarrow 0
$$

which is split because $\mathbb{k}$ is contained as a $\mathbb{k}$-subalgebra of $A_{\mathbb{k}}(S ; \mathcal{F})$ generated by the trivial $S$-set $*$. Applying this to the trivial fusion system over $S$ we obtain a short exact sequence

$$
0 \rightarrow \widehat{I_{\mathbb{k}}}(S) \rightarrow \widehat{A_{\mathbb{k}}}(S) \stackrel{\hat{\epsilon}}{\rightarrow} \mathbb{k} \rightarrow 0 .
$$

The inclusions $A_{\mathbb{k}}(S ; \mathcal{F}) \subseteq A_{\mathbb{k}}(S)$ and $I_{\mathbb{k}}(S ; \mathcal{F}) \subseteq I_{\mathbb{k}}(S)$ yield a ring homomorphism $\widehat{A_{\mathbb{k}}}(S ; \mathcal{F}) \rightarrow \widehat{A_{k}}(S)$ which factors through

$$
\Psi: \widehat{A_{\mathbb{k}}}(S ; \mathcal{F}) \rightarrow \widehat{A_{\mathbb{k}}}(S)^{\mathcal{F}}
$$

because $A_{\mathbb{k}}(S ; \mathcal{F}) \subseteq A_{\mathbb{k}}(S)^{\mathcal{F}}$ by Proposition 3.6.

By definition $\widehat{I_{\mathrm{k}}}(S)^{\mathcal{F}}=\widehat{I}_{\mathbb{k}}(S) \cap \widehat{A_{\mathbb{k}}}(S)^{\mathcal{F}}$ and therefore from (4.11) we now obtain the following morphism of short exact sequences

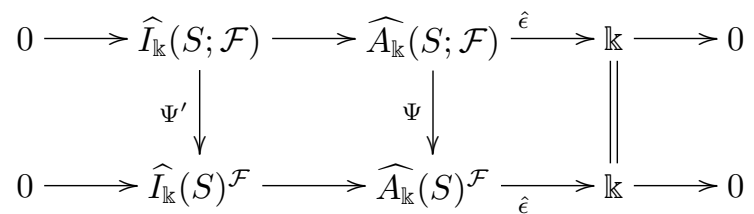

By Proposition 4.3 and from the fact that $I_{\mathbb{k}}(S ; \mathcal{F})$ is a finitely generated free $\mathbb{k}$ module by Lemma 4.2 , we see that

$$
\begin{aligned}
& \widehat{I}_{\mathbb{k}}(S ; \mathcal{F}) \cong I_{\mathbb{k}}(S ; \mathcal{F})_{p}^{\wedge} \cong I_{\mathbb{k}}(S ; \mathcal{F}) \otimes_{\mathbb{k}} \mathbb{k}_{p}^{\wedge}=I_{\mathbb{k}_{p}}(S ; \mathcal{F}), \\
& \widehat{I}_{\mathbb{k}}(S) \cong I_{\mathbb{k}}(S)_{p}^{\wedge} \cong I_{\mathbb{k}}(S) \otimes_{\mathbb{k}} \mathbb{k}_{p}^{\wedge}=I_{\mathbb{k}_{p}}(S) .
\end{aligned}
$$

Thus, $\Psi^{\prime}$ is the map

$$
I_{\mathbb{k}_{\hat{p}}}(S ; \mathcal{F}) \subseteq I_{\mathbb{k}_{p}}(S)^{\mathcal{F}}
$$

induced by the inclusion $I(S ; \mathcal{F}) \subseteq I(S)^{\mathcal{F}}=A(S)^{\mathcal{F}} \cap I(S)$. By Proposition 3.6 and Lemma 4.2, this inclusion is in fact an equality and therefore $\Psi^{\prime}$ is an isomorphism. This proves point (1) of this Proposition. The five-lemma now shows that $\Psi$ is an isomorphism and this is point (2). The first assertion of point (3) follows from the 
first row in the commutative ladder above. The second assertion follows because if $\mathbb{k}$ is $p$-complete, namely $\mathbb{k}_{p}^{\wedge}=\mathbb{k}$, then $\widehat{I_{\mathbb{k}}}(S ; \mathcal{F})=I_{\mathbb{k}}(S ; \mathcal{F})$.

Proof of Theorem B. This follows from Lemma 4.7 and Propositions 4.9 and 3.6. For part (2) of the theorem we also need Lemma 4.2 .

Proof of Corollary $C$. In the presence of a $p$-local finite group, the canonical map $f: B S \rightarrow|\mathcal{L}|_{p}^{\wedge}$ induces $\omega_{\mathcal{F}}$. By Theorem $\mathrm{B}$, it gives rise to a ring monomorphism $f^{*}: \pi^{0}\left(|\mathcal{L}|_{p}^{\wedge} \vee \mathbb{S}\right) \stackrel{\pi^{0}(f \vee \mathbb{S})}{\longrightarrow} \pi^{0}(B S \vee \mathbb{S}) \cong \widehat{A}(S)$ whose image is $\widehat{A}(S)^{\mathcal{F}} \cong\left(A(S)^{\mathcal{F}}\right)_{I}^{\wedge}$ Finally, we note that $\pi^{0}(f \vee \mathbb{S})$ can be replaced with $\pi^{0}\left(|\mathcal{L}|_{p_{+}}^{\wedge}\right) \stackrel{\pi^{0}\left(f_{+}\right)}{\longrightarrow} \pi^{0}\left(B S_{+}\right)$, see Remark 4.6.

In the next result we obtain some information on $\mathbb{Z}_{(p)} \otimes \pi^{0}(\mathbb{B} \mathcal{F} \vee \mathbb{S})$.

4.12. Proposition. Consider the rings $R_{1}=\mathbb{Z}_{(p)} \otimes \widehat{A}(S ; \mathcal{F})$ and $R_{2}=A_{(p)}(S ; \mathcal{F})$. Then

(1) Both rings are local with residue field isomorphic to $\mathbb{F}_{p}$.

(2) Their non-maximal prime ideals are in one-to-one correspondence with the $\mathcal{F}$-conjugacy classes $[H]$ of the subgroups of $S$ and we denote them by $\hat{\mathfrak{p}}_{[H], 0} \triangleleft R_{1}$ and $\mathfrak{p}_{[H], 0} \triangleleft R_{2}$. Each one of these ideals is contained only in the maximal ideal.

(3) The quotient rings $R_{2} / \mathfrak{p}_{[H], 0}$ are isomorphic to $\mathbb{Z}_{(p)}$. The quotient rings $R_{1} / \hat{\mathfrak{p}}_{[H], 0}$ are isomorphic to $\mathbb{Z}_{p}^{\wedge}$ if $H \neq 1$ and to $\mathbb{Z}_{(p)}$ if $H=1$.

Proof. To avoid triviality we assume that $S \neq 1$. Set $R=A_{(p)}(S ; \mathcal{F})$ and $I=$ $I_{(p)}(S ; \mathcal{F})$. By $(4.11)$ the inclusion $A(S ; \mathcal{F}) \subseteq A_{(p)}(S ; \mathcal{F})$ induces a morphism of short exact sequences

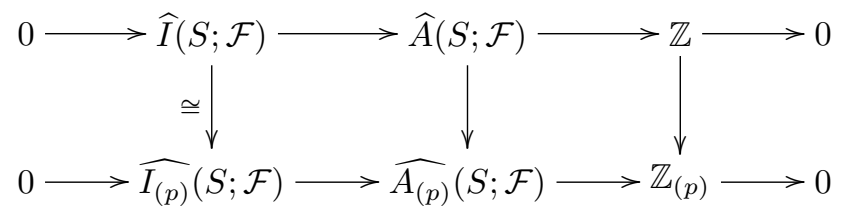

and by Proposition 4.9 the vertical arrow on the left is an isomorphism because

$$
\widehat{I}(S ; \mathcal{F}) \cong I_{\mathbb{Z}_{p}^{\wedge}}(S ; \mathcal{F}) \cong I_{\mathbb{Z}_{(p)} \wedge}(S ; \mathcal{F}) \cong \widehat{I_{(p)}}(S ; \mathcal{F})
$$

It follows that

$$
R_{1}=\mathbb{Z}_{(p)} \otimes \widehat{A}(S ; \mathcal{F})=\widehat{A_{(p)}}(S ; \mathcal{F})=R_{I}^{\wedge}
$$

Our goal now is to study the prime ideals in $\hat{R}:=R_{I}^{\wedge}$. We will write $\hat{I}$ for the $I$-completion of $I$ and we note that this is an ideal of $\hat{R}$. By Theorem $2.9 \hat{R}$ is $\hat{I}$-complete.

Observe that $R$ is a subring of $\hat{R}$ because the $I$-adic topology on $R$ is Hausdorff because the $I$-adic topology on $I$ is equivalent to the $p$-adic topology and $I$ is a free $\mathbb{Z}_{(p)}$-module. Also note that $R$ is a Noetherian ring because it is a finitely generated $\mathbb{Z}_{(p)}$-module. As a consequence $\hat{R}$ is also Noetherian by Theorem 2.7.

Fix a prime ideal $\hat{\mathfrak{p}} \triangleleft \hat{R}$. By Theorem 2.8 and the fact that $\hat{R}$ is $\hat{I}$-complete, we deduce that $\hat{R} / \hat{\mathfrak{p}}$ is $\hat{I}$-complete as an $\hat{R}$-module. However, the $\hat{I}$-adic topology on $\hat{R} / \hat{\mathfrak{p}}$ is equivalent to the $I$-adic topology by Theorem 2.9 . We now deduce that $\hat{R} / \hat{\mathfrak{p}}$ is $I$-complete as an $R$-module. 
Recall from Proposition 3.6 that $R$ has a $\mathbb{Z}_{(p)}$-basis $\left\{\Omega_{Q_{i}}\right\}$ where $Q_{i}$ are representatives for the $\mathcal{F}$-conjugacy classes of the subgroups of $S$. Let $\pi: \hat{R} \rightarrow \hat{R} / \hat{\mathfrak{p}}$ denote the quotient map and note that $R \subseteq \hat{R}$. Choose $H=Q_{t}$ of minimal order such that $\pi\left(\Omega_{H}\right) \neq 0$. For any $K=Q_{j}$ we know that all the isotropy groups of $\Omega_{H} \times \Omega_{K}$ are $\mathcal{F}$-conjugate to subgroups of $H$. Therefore, by Proposition 3.6

$$
\Omega_{H} \times \Omega_{K}=\left|\left(\Omega_{K}\right)^{H}\right| \cdot \Omega_{H}+\sum_{j} \alpha_{j} \cdot \Omega_{L_{j}},
$$

where $L_{j}$ are $\mathcal{F}$-conjugate to proper subgroups of $H$, whence $\pi\left(\Omega_{L_{j}}\right)=0$ by the minimality of $|H|$. It follows that $\pi\left(\Omega_{H}\right) \cdot \pi\left(\Omega_{K}\right)=\left|\left(\Omega_{K}\right)^{H}\right| \cdot \pi\left(\Omega_{H}\right)$. Since $\pi\left(\Omega_{H}\right) \neq$ 0 and $\hat{R} / \hat{\mathfrak{p}}$ is an integral domain we deduce that

$$
\pi\left(\Omega_{K}\right)=\left|\left(\Omega_{K}\right)^{H}\right| \cdot 1_{\hat{R} / \hat{\mathfrak{p}}}=\chi_{H}\left(\Omega_{K}\right) \cdot 1_{\hat{R} / \hat{\mathfrak{p}}} .
$$

Thus, the following square in the category of $R$-modules is commutative

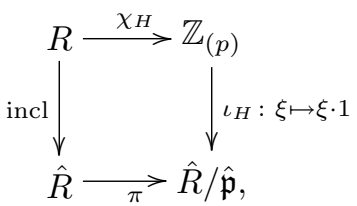

where the $R$-module structure on $\mathbb{Z}_{(p)}$ is induced by $\chi_{H}$. Since $\hat{R} / \hat{\mathfrak{p}}$ is $I$-complete, $\pi$ is equal to the composite

$$
\hat{R} \stackrel{\hat{\chi}_{H}}{\longrightarrow}\left(\mathbb{Z}_{(p)}\right)_{I}^{\wedge} \stackrel{\left(\iota_{H}\right)_{I}^{\wedge}}{\longrightarrow} \hat{R} / \hat{\mathfrak{p}} .
$$

Now, if $H=1$ then the $I$-adic topology on $\mathbb{Z}_{(p)}$ is the discrete one because $\epsilon=\chi_{(1)}$ and if $H \neq 1$ then it is the $p$-adic topology because $\chi_{H}(S / e-|S|) \neq 0$. Since $\pi$ is surjective, we deduce that $\hat{R} / \hat{\mathfrak{p}}$ is either $\mathbb{Z}_{(p)}$ or $\mathbb{Z}_{p}^{\wedge}$ or it is $\mathbb{F}_{p}$.

Assume first that $\hat{R} / \hat{\mathfrak{p}}=\mathbb{F}_{p}$. Now, $\chi_{H}\left(\Omega_{H}\right)=0 \bmod p$ if $H \lesseqgtr S$ by Proposition 3.3 , so from the way $H$ was chosen we deduce that $H=S$. Therefore $\hat{\mathfrak{p}}$ is the kernel of

$$
\hat{R} \stackrel{\hat{\chi}_{S}}{\longrightarrow} \mathbb{Z}_{p}^{\wedge} \rightarrow \mathbb{F}_{p}
$$

and it follows that $\hat{R}$ is a local ring. Now assume that $\operatorname{char}(\hat{R} / \hat{\mathfrak{p}})=0$. In this case, $\hat{R} / \hat{\mathfrak{p}} \cong\left(\mathbb{Z}_{(p)}\right)_{I}^{\wedge}$ and so $\hat{\mathfrak{p}}$ is the kernel of

$$
\begin{array}{ll}
\hat{\chi}_{H}: \hat{R} \rightarrow \mathbb{Z}_{p}^{\wedge} & \text { if } H \neq 1 \text { or } \\
\hat{\chi}_{1}: \hat{R} \rightarrow \mathbb{Z}_{(p)} & \text { if } H=1 .
\end{array}
$$

We denote these kernels by $\hat{\mathfrak{p}}_{[H], 0}$ which are clearly prime ideals. It only remains to show that these ideals are distinct and that none is contained in the other.

Suppose that $\hat{\mathfrak{p}}_{[H], 0} \subseteq \hat{\mathfrak{p}}_{[K], 0}$. Then we get a surjection $\hat{R} / \hat{\mathfrak{p}}_{[H], 0} \rightarrow \hat{R} / \hat{\mathfrak{p}}_{[K], 0}$ where both rings are isomorphic to either $\mathbb{Z}_{(p)}$ or $\mathbb{Z}_{p}^{\wedge}$, whence this surjection must be an isomorphism and therefore $\hat{\mathfrak{p}}_{[H], 0}=\hat{\mathfrak{p}}_{[K], 0}$. Now suppose that $H=Q_{i}$ and $K=Q_{j}$ are not $\mathcal{F}$-conjugate. Without loss of generality we may assume that $H$ is not $\mathcal{F}$-conjugate to a subgroup of $K$ whence $\left(\Omega_{K}\right)^{H}=\emptyset$. This shows that $\Omega_{K} \in \operatorname{ker}\left(\hat{\chi}_{H}\right)=\hat{\mathfrak{p}}_{[H], 0}$. However $\hat{\chi}_{K}\left(\Omega_{K}\right)=\left|\frac{N_{S} K}{K}\right| \neq 0$ so $\Omega_{K} \notin \hat{\mathfrak{p}}_{[K], 0}$. Thus, $\hat{\mathfrak{p}}_{[H], 0}$ and $\hat{\mathfrak{p}}_{[K], 0}$ are distinct. This completes the analysis of the spectrum of the prime ideals in $R_{1}=\hat{R}$. 
The analysis of the spectrum of the prime ideals in $R_{2}=R$ is similar. Let $\mathfrak{p}_{[H], 0}$ be the kernel of $\chi_{H}: R \rightarrow \mathbb{Z}_{(p)}$ and let $\mathfrak{m}$ denote the kernel of $\chi_{S}: R \rightarrow \mathbb{Z}_{(p)} \rightarrow \mathbb{F}_{p}$. Consider a prime ideal $\mathfrak{p} \triangleleft R$ ad let $\pi: R \rightarrow R / \mathfrak{p}$ denote the quotient map. Set $H=Q_{t}$ where $H$ has minimal order with the property that $\pi\left(\Omega_{H}\right) \neq 0$. One argues as above to prove that $\pi$ may be identified with

$$
R \stackrel{\chi_{H}}{\longrightarrow} \mathbb{Z}_{(p)} \quad \text { or } \quad R \stackrel{\chi_{H}}{\longrightarrow} \mathbb{Z}_{(p)} \rightarrow \mathbb{F}_{p} .
$$

If the second possibility happens then $H=S$ because $\pi\left(\Omega_{H}\right)=\chi_{H}\left(\Omega_{H}\right)=\left|\frac{N_{S} H}{H}\right|=$ $0 \bmod p$ if $H \leq S$. We therefore see that $\mathfrak{m}$ is the unique maximal ideal in $R$. If $R / \mathfrak{p} \cong \mathbb{Z}_{(p)}$ then $\mathfrak{p}=\mathfrak{p}_{[H], 0}$ and the argument above for $\hat{\mathfrak{p}}_{[H], 0}$ shows that the ideals $\mathfrak{p}_{[K], 0}$ are distinct for non- $\mathcal{F}$-conjugate $K$ 's and that none is contained in the other.

\section{REFERENCES}

[1] J. F. Adams. Stable homotopy and generalised homology. Chicago Lectures in Mathematics. University of Chicago Press, 1974.

[2] C. Broto, R. Levi, B. Oliver. The homotopy theory of fusion systems. J. Amer. Math. Soc. 16 (2003), no. 4, 779-856

[3] Diaz A., Libman A. The Burnside ring of saturated fusion systems. Available from http://www.maths.abdn.ac.uk/ bensondj/html/archive/diaz-libman.html

[4] D. Eisenbud, Commutative algebra. With a view toward algebraic geometry. Graduate Texts in Mathematics, 150. Springer-Verlag, New York, 1995.

[5] S. Jackowski, J. McClure. Homotopy decomposition of classifying spaces via elementary abelian subgroups. Topology 31 (1992), no. 1, 113-132.

[6] J. P. May, J. E. McClure, A reduction of the Segal conjecture. Current trends in algebraic topology, Part 2 (London, Ont., 1981), pp. 209-222, CMS Conf. Proc., 2, Amer. Math. Soc.

[7] Ll. Puig, Frobenius categories. J. Algebra 303 (2006), no. 1, 309-357.

[8] K. Ragnarsson, A Segal conjecture for $p$-completed classifying spaces. Adv. Math. 215 (2007), no. $2,540-568$

[9] K. Ragnarsson, Classifying spectra of saturated fusion systems. Algebr. Geom. Topol. 6 (2006), 195-252

(Antonio Díaz) Department of Mathematical Sciences, University of Copenhagen, Universitetsparken 5, DK-2100 Copenhagen, Denmark

(Assaf Libman) Department of Mathematical Sciences, King's College, University of Aberdeen, Aberdeen AB24 3UE, Scotland, U.K. 